Publications of the Astronomical Society of the Pacific, 117:1255-1262, 2005 November

(C) 2005. The Astronomical Society of the Pacific. All rights reserved. Printed in U.S.A.

\title{
A Data Exchange Standard for Optical (Visible/IR) Interferometry
}

\author{
T. A. Pauls \\ Naval Research Laboratory, Code 7210, 4555 Overlook Avenue SW, Washington, DC 20375-5351; pauls@nrl.navy.mil \\ J. S. Young \\ Astrophysics Group, Cavendish Laboratory, Madingley Road, CB3 0HE, UK; jsy1001@cam.ac.uk \\ W. D. CotTon \\ NRAO, 520 Edgemont Road, Charlottesville, VA 22903; bcotton@nrao.edu \\ AND \\ J. D. MONnier \\ Department of Astronomy, University of Michigan, Ann Arbor, MI 48109; monnier@umich.edu \\ Received 2005 June 30; accepted 2005 July 7; published 2005 September 28
}

\begin{abstract}
This paper describes the OI (Optical Interferometry) Exchange Format, a standard for exchanging calibrated data from optical (visible/infrared) stellar interferometers. The standard is based on the Flexible Image Transport System (FITS) and supports the storage of optical interferometric observables, including squared visibility and closure phase-data products not included in radio interferometry standards such as UV-FITS. The format has already gained the support of most currently operating optical interferometer projects, including COAST, NPOI, IOTA, CHARA, VLTI, PTI, and the Keck Interferometer, and is endorsed by the IAU Working Group on Optical Interferometry. Software is available for reading, writing, and the merging of OI Exchange Format files.
\end{abstract}

\section{INTRODUCTION}

The idea of a common data format for visible wavelength and infrared astronomical interferometry (henceforth referred to collectively as "optical interferometry") arose through discussions at the 2000 June NSF-sponsored meeting in Socorro, New Mexico (McAlister \& Cornwell 2000). Since 2001, T. P. and J. S. Y. have been responsible, under the auspices of the International Astronomical Union (IAU), for coordinating discussion on this topic, and for producing and maintaining the specification document for a format based on the Flexible Image Transport System (FITS; Hanisch et al. 2001).

\subsection{Motivation}

The motivation for a creating a data format specific to optical interferometry was twofold. First, existing formats designed for radio interferometry, such as UV-FITS (Cotton et al. 1990) and FITS-IDI (Flatters 2000), do not describe optical data adequately. Second, the more popular UV-FITS format is poorly defined-its content has evolved, and the format is partly defined by reference to the behavior of the AIPS software-and is based on the deprecated FITS "Random Group" conventions.

\footnotetext{
${ }^{1}$ We request that comments and suggestions related to the OI Exchange Format be directed to the OLBIN e-mail list (see http://listes.obs.ujf-grenoble.fr/wws/ info/olbin for information on how to subscribe and post to the list).
}

The principal shortcoming of the radio interferometry formats is that Fourier data are stored as complex visibilities. The noise models for radio and optical interferometry are quite different; optical interferometers must usually integrate incoherently to obtain useful signal-to-noise ratios. Thus, the "good" observables in the optical are calibrated fringe power spectra (squared visibility amplitudes) and bispectra (tripleproduct amplitudes and closure phases), rather than complex visibilities. A file format based on complex visibilities can only encode (optical) closure phases if corresponding visibility amplitude and closure-phase measurements are made simultaneously, and even then the uncertainties on the closure phases are not stored (radio interferometry analysis software assumes zero closure-phase error). There is also no way of saving bispectrum (triple product) amplitudes.

\subsection{History}

Preliminary drafts of the specification for an optical interferometry format were discussed by the community at the 198th Meeting of the American Astronomical Society in 2001 June and the 2001 August Meeting of the IAU Working Group on Optical/IR Interferometry. The discussion continued by e-mail among various interested parties, and the document was revised.

A public prerelease of the format specification and accom- 
TABLE 1

History of the OI Exchange Format

\begin{tabular}{cl}
\hline \hline Date & \\
\hline 2002 Mar $25 \ldots \ldots$ & Prerelease of document and example code; release 1 of format specification. \\
2002 Apr $25 \ldots \ldots$ & Minor correction to document; release 2 of format specification. \\
2002 Nov $26 \ldots \ldots$ & Post-IAU-WG meeting release of document and example; release 3 of format specification. \\
2003 Feb $17 \ldots \ldots$. & Release of document, C, and IDL code to fix problem in OI_TARGET table; release 4 of format specification. \\
2003 Apr $7 \ldots \ldots \ldots$ & Freeze of format specification. Revision numbers of all tables set to unity; release 5 of format specification. \\
$2005 \ldots \ldots \ldots \ldots$. & This paper; no changes to exchange format or table revision numbers. \\
\hline \hline
\end{tabular}

panying example $\mathrm{C}$ code was made in 2002 March, followed by a second prerelease of the document in 2002 April. This draft was discussed at the 2002 August IAU Working Group meeting. Comments were presented by participants from the European Southern Observatory and the Interferometry Science Center (now the Michelson Science Center). Following the IAU Working Group meeting, there were two further prereleases of the format specification prior to the first "production" release.

The standard was frozen on 2003 April 7 (release 5 of the format specification), meaning that subsequent changes would require increments of the revision numbers for the changed tables. The history of the format specification is summarized in Table 1.

The standard is now supported by the majority of optical interferometer projects, including COAST, NPOI, IOTA, CHARA, VLTI, PTI, and the Keck Interferometer.

\subsection{OIFITS Publications}

This paper contains the formal definition of the standard, beginning in $\S 3$. As earlier versions of the format specification have only been distributed via the World Wide Web, this paper formalizes the standard and serves as a published reference for it. Further discussion that explains the design decisions and provides explicit pointers to existing software for reading and writing the format can be found in Pauls et al. (2004), which is intended as a "companion" to the official format specification given here.

A first application of the Exchange Format, a "beauty contest" to compare the performance of a number of image reconstruction software packages, is described by Lawson et al. (2004). The contest demonstrated that four different international groups could faithfully reconstruct images from simulated data sets in the OIFITS format.

No alterations have been made to the standard since release 5 of the format specification. The text in $\S 3$ onward is substantially the same as that in release 5, apart from minor changes to clarify certain points and to conform to journal style. The revision numbers of all tables defined by the standard remain at unity.

\section{PURPOSE AND SCOPE}

By defining and maintaining a common data format, we hope to encourage the development of common software for the analysis of data from optical stellar interferometers, and to facilitate the exchange of data between different research groups. In this way, the limited Fourier coverage of current instruments can be ameliorated by combining data from several interferometers. An example of this is given in Monnier et al. (2004).

The format is intended to support the storage of calibrated, time-averaged data. Such data can be prepared from the raw fringe measurements without using information about the detailed structure of the target (i.e., without doing any astrophysical interpretation), yet once the data is in the format, it can be analyzed without knowing the details of the instrument. Calibrated data from different interferometers can be treated in the same way (provided there are no residual systematic errors).

The standard includes the information needed to perform "imaging" from a calibrated data set. Here imaging refers loosely to any process for making inferences about the skybrightness distribution from the data. The standard explicitly allows extra binary tables or columns, beyond those it defines, to be used to store additional information. In this way, the standard can be used for other purposes besides imaging (e.g., for astrometry or as an archive format).

We suggest that the common data format be referred to as the "OI Exchange Format," or the "Exchange Format" when the context is clear. A suitable very short moniker is "OIFITS" (or "OI-FITS").

\section{DOCUMENT CONVENTIONS}

In what follows we use the FITS binary table nomenclature of keywords and column headings. The values associated with the keywords can be considered as scalars, while each column can be simply an array or an array of "pointers" to other arrays. The following data types are used in the standard: $\mathrm{I}=$ integer (16 bit), $\mathrm{A}=$ character, $\mathrm{E}=$ real $(32 \mathrm{bit}), \mathrm{D}=$ double $(64$ bit), $\mathrm{L}=$ logical. In the tables below, the number in parentheses is the dimensionality of the entry. The table names given below correspond to the values of the EXTNAME keyword. Other mandatory keywords describing the structure of the FITS binary tables (see Hanisch et al. 2001) have been omitted from this document. Hanisch et al. (2001) also describes various extensions to binary tables that are not part of the FITS standard. None of these are currently used in this format. The definitions of all tables have been "frozen" since 2003 April. 


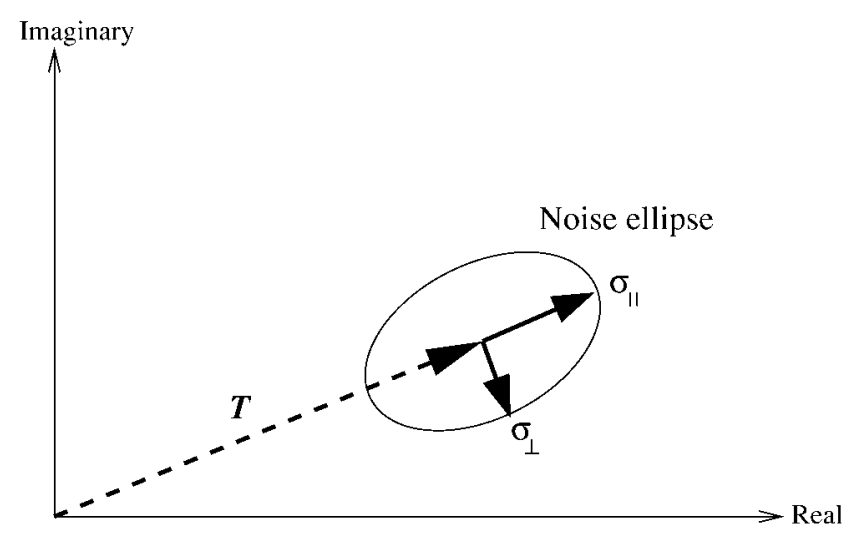

FIG. 1.-Noise model for triple product. The figure is an Argand diagram showing the mean triple product $T$ with its associated noise ellipse, elongated parallel to $T$. See text for explanation.

All revision numbers are currently equal to 1 . Any future changes will require increments in the revision numbers of the altered tables.

\section{DEFINITIONS AND ASSUMPTIONS}

The conventions described here are generally identical to those used in radio interferometry and described in standard textbooks (e.g., Thompson et al. 1986).

\subsection{Baseline Vector}

The baseline vector between two interferometric stations A and $\mathrm{B}$, whose position vectors are $\boldsymbol{x}_{\mathrm{A}}$ and $\boldsymbol{x}_{\mathrm{B}}$, is defined as $\boldsymbol{A B}=\boldsymbol{x}_{\mathrm{B}}-\boldsymbol{x}_{\mathrm{A}}$.

\section{2. $u v$ Coordinates}

The coordinate $u$ is the east component and $v$ is the north component of the projection of the baseline vector onto the plane normal to the direction of the phase center, which is assumed to be the pointing center.

\subsection{Complex Visibility}

The basic observable of an interferometer is the complex visibility, which is related to the sky-brightness distribution $I(x, y)$ by a Fourier transform:

$$
V(u, v)=\iint d x d y I(x, y) \exp [-2 \pi i(u x+v y)],
$$

where $x$ and $y$ are displacements (in radians) in the plane of the sky (which is assumed to be flat). The origin of this coordinate system is the phase center, and $x$ is in the direction of increasing right ascension (i.e., the $x$ axis points east), while $y$ is in the direction of increasing declination (the $y$ axis points north). With $x$ and $y$ defined, the above equation defines the sign convention for complex visibilities that should be used in the data format. The visibility is normalized by the total flux from the target, which is assumed to remain constant over the time spanned by the measurements in the file. Neither the field of view over which the "total" flux is collected nor the field of view over which fringes are detected (i.e., the limits of the above integral) can be inferred from the data file.

\subsection{Squared Visibility}

The squared visibility is simply the modulus squared of the complex visibility:

$$
S(u, v)=|V(u, v)|^{2} .
$$

\subsection{Triple Product}

The triple product (strictly the bispectrum) is the product of the complex visibilities on three baselines, which form a closed loop joining three stations $\mathrm{A}, \mathrm{B}$, and $\mathrm{C}$. In the following expression, $\left(u_{1}, v_{1}\right)$ is the projection of $\mathrm{AB}$, and $\left(u_{2}, v_{2}\right)$ is the projection of $\mathrm{BC}$ [and hence $\left(u_{1}+u_{2}, v_{1}+v_{2}\right)$ is the projection of $\mathrm{AC}]$ :

$$
\begin{aligned}
T\left(u_{1}, v_{1}, u_{2}, v_{2}\right) & =V\left(u_{1}, v_{1}\right) V\left(u_{2}, v_{2}\right) \\
& \times V^{*}\left(u_{1}+u_{2}, v_{1}+v_{2}\right) .
\end{aligned}
$$

\subsection{Noise Model for Triple Product}

The data are assumed to be complex triple products averaged over a large number of "exposures." In such a case, the noise can be fully described in terms of a Gaussian noise ellipse in the complex plane. Photon, detector, and background noise tend to lead to noise ellipses that are close to circular. On the other hand, fluctuating atmospheric phase errors across telescope apertures typically cause fluctuations in the amplitude of the triple product that are much larger than the fluctuations in the phase. Thus, the "atmospheric" contribution to the noise ellipse is elongated along the direction of the mean triple-product vector in the Argand diagram, as shown in Figure 1. Such noise needs to be characterized in terms of the variance $\sigma_{\perp}^{2}$ perpendicular to the mean triple-product vector and the variance $\sigma_{\|}^{2}$ parallel to $T$. We can parameterize the perpendicular variance in terms of a "phase error" $\sigma_{\theta}=(180 / \pi)\left(\sigma_{\perp} /|T|\right)$. The phase error gives an approximate value for the rms error in the closure phase, in degrees. We denote $\sigma_{\|}$as the "amplitude error."

In many cases, the observer may be interested primarily in the closure phase and not the triple-product amplitude, and therefore may choose not to calibrate the amplitude. Such a case can be indicated in the above notation as an infinite amplitude error and a finite phase error. The data format specifies that such a case should be indicated by a NULL value for the amplitude (the amplitude error value is then ignored). 
TABLE 2

OI_ARRAY (REVISION 1)

\begin{tabular}{|c|c|c|}
\hline Label & Data Type & Description \\
\hline \multicolumn{3}{|c|}{ Keywords } \\
\hline OI_REVN ...... & $\mathrm{I}$ & Revision number of the table definition \\
\hline ARRNAME $\ldots \ldots \ldots \ldots \ldots \ldots \ldots \ldots \ldots$ & A & Array name, for cross-referencing \\
\hline FRAME $\ldots \ldots \ldots \ldots \ldots \ldots \ldots \ldots \ldots$ & A & Coordinate frame \\
\hline ARRAYX, ARRAYY, ARRAYZ ...... & $\mathrm{D}$ & Array center coordinates (m) \\
\hline \multicolumn{3}{|c|}{ Column Headings (one row for each type) } \\
\hline TEL_NAME $\ldots \ldots \ldots \ldots \ldots \ldots \ldots \ldots$ & A (16) & Telescope name \\
\hline STA_NAME $\ldots \ldots \ldots \ldots \ldots \ldots \ldots \ldots$ & A (16) & Station name \\
\hline STA_INDEX $\ldots \ldots \ldots \ldots \ldots \ldots \ldots \ldots$ & I (1) & Station number \\
\hline DIAMETER $\ldots \ldots \ldots \ldots \ldots \ldots \ldots \ldots$ & $\mathrm{E}(1)$ & Element diameter $(\mathrm{m})$ \\
\hline STAXYZ $\ldots \ldots \ldots \ldots \ldots \ldots \ldots \ldots \ldots$ & $\mathrm{D}(3)$ & Station coordinates relative to array center $(\mathrm{m})$ \\
\hline
\end{tabular}

\subsection{Noise Model for Complex Visibility}

There was much discussion on the (now defunct) oi-data@ rsd.nrl.navy.mil e-mail list concerning which representation to use for complex visibilities in the standard. A number of different classes of data can be represented as complex visibilities, including several varieties of differential phase data. In all cases, the standard should only be used to store averaged data. Thus, as with triple products, we must consider the shape of the noise ellipse in the complex plane.

It has been demonstrated (Hummel et al. 2002) that both circularly symmetric noise and noise ellipses elongated parallel to or perpendicular to the mean vector can occur, in practice. Thus far, there has been no evidence for noise ellipses elongated parallel to the real or imaginary axes, although examples of some classes of data have yet to be presented. Hence, an amplitude/phase representation of complex visibilities, mirroring that used for triple products, has been adopted in the current version of the standard.

\section{FITS FILE STRUCTURE}

A valid exchange-format FITS file must contain one (and only one) OI_TARGET table, plus one or more of the data tables: OI_VIS, OI_VIS2, or OI_T3. Each data table must refer to an OI_WAVELENGTH table that is present in the file. There may be more than one of each type of data table (e.g., OI_VIS2). One or more OI_ARRAY tables (or equivalent; e.g., for aperture masking in future releases of the standard) may optionally be present. Where multiple tables of the same EXTNAME are present, each should have a unique value of EXTVER (this according to the FITS standard; however, the example C code and J. D. M.'s IDL software do not require EXTVER to be present).

The tables can appear in any order. Other header/data units may appear in the file, provided their EXTNAMEs do not begin with "OI_". Reading software should not assume that either the keywords or the columns in a table appear in a particular order. This is straightforward to implement using software libraries such as CFITSIO.
Any of the tables may have extra keywords or columns beyond those defined in the standard. It would facilitate the addition of new keywords and columns in future releases of the standard if the nonstandard keywords and column names were given a particular prefix (e.g., "NS_") to avoid conflicts.

\section{TABLES DEFINED BY THE STANDARD}

The specifications for the OI_ARRAY table are given in Table 2. As defined, this table is aimed at ground-based interferometry with separated telescopes.

\subsection{OI_ARRAY (Revision 1) Specifications}

Alternative tables could be used for other cases. These must have at least an ARRNAME keyword, for cross-referencing purposes. Each OI_ARRAY-equivalent table in a file must have a unique value for ARRNAME.

\subsubsection{Number of Elements}

There is no keyword giving the number of elements (NELEMENT in a previous revision of this document), as this is equal to the number of rows in the FITS binary table, which is given by the standard NAXIS2 keyword. For the same reason, there are no format-specific keywords giving the number of rows in any of the other tables.

\subsubsection{Coordinate Frame}

If the FRAME keyword has the value "GEOCENTRIC," then the coordinates are given in an Earth-centered, Earth-fixed, Cartesian reference frame. The origin of the coordinates is the Earth's center of mass. The $z$ axis is parallel to the direction of the conventional origin for polar motion. The $x$ axis is parallel to the direction of the intersection of the Greenwich meridian with the mean astronomical equator. The $y$ axis completes the right-handed, orthogonal coordinate system.

Currently, no other values for the FRAME keyword may be used. This will change if the need arises. 
TABLE 3

OI_TARGET (REVISION 1)

\begin{tabular}{|c|c|c|}
\hline Label & Data Type & Description \\
\hline \multicolumn{3}{|r|}{ Keywords } \\
\hline OI_REVN $\ldots \ldots \ldots \ldots$ & $\mathrm{I}$ & Revision number of the table definition \\
\hline \multicolumn{3}{|c|}{ Column Headings (one row for each source) } \\
\hline TARGET_ID ....... & I $(1)$ & Index number \\
\hline TARGET $\ldots . . . \ldots$. & A (16) & Target name \\
\hline RAEP0 $\ldots \ldots \ldots \ldots$ & $\mathrm{D}(1)$ & R.A. at mean equinox (deg) \\
\hline DECEP0 .......... & $\mathrm{D}(1)$ & Decl. at mean equinox (deg) \\
\hline EQUINOX $\ldots . . . .$. & E (1) & Equinox \\
\hline RA_ERR $\ldots . . . \ldots .$. & $\mathrm{D}(1)$ & Error in R.A. at mean equinox (deg) \\
\hline DEC_ERR .......... & $\mathrm{D}(1)$ & Error in decl. at mean equinox (deg) \\
\hline SYSVEL $\ldots \ldots \ldots \ldots$ & $\mathrm{D}(1)$ & Systemic radial velocity $\left(\mathrm{m} \mathrm{s}^{-1}\right)$ \\
\hline VELTYP . .......... & A (8) & Reference for radial velocity (LSR, GEOCENTR, etc.) \\
\hline VELDEF $\ldots \ldots \ldots \ldots$ & A (8) & Definition of radial velocity (OPTICAL, RADIO) \\
\hline PMRA $\ldots \ldots \ldots \ldots \ldots$ & $\mathrm{D}(1)$ & Proper motion in R.A. $\left(\mathrm{deg} \mathrm{yr}^{-1}\right)$ \\
\hline PMDEC $\ldots \ldots \ldots \ldots$ & $\mathrm{D}(1)$ & Proper motion in decl. $\left(\operatorname{deg} \mathrm{yr}^{-1}\right)$ \\
\hline PMRA_ERR ....... & $\mathrm{D}(1)$ & Error of proper motion in R.A. (deg $\mathrm{yr}^{-1}$ ) \\
\hline PMDEC_ERR ...... & $\mathrm{D}(1)$ & Error of proper motion in decl. $\left(\mathrm{deg} \mathrm{yr}^{-1}\right)$ \\
\hline PARALLAX $\ldots \ldots$. & $\mathrm{E}(1)$ & Parallax (deg) \\
\hline PARA_ERR...... & $\mathrm{E}(1)$ & Error in parallax (deg) \\
\hline SPECTYP . . ....... & A (16) & Spectral type \\
\hline
\end{tabular}

\subsubsection{Array Coordinates}

The ARRAYX, ARRAYY, and ARRAYZ keywords give the coordinates of the array center in the coordinate frame specified by the FRAME keyword. Element coordinates in the main part of the table are given relative to the array center, in the same coordinate frame. Coordinates are given in meters.

\subsubsection{Station Number}

Each row in the table is assigned a unique station number, which is used in other tables as an index into this one. The table structure is the simplest possible; i.e., there is no explicit concept of different "configurations" within the table. Each row in the table corresponds to a distinct set of station coordinates used in taking the data stored in the file.

\subsubsection{Element Diameter}

This is the effective aperture size; e.g., if the telescope is stopped down.

TABLE 4

VELTYP STRINGS

\begin{tabular}{ll}
\hline \hline \multicolumn{1}{c}{ String } & \multicolumn{1}{c}{ Description } \\
\hline LSR $\ldots \ldots \ldots \ldots \ldots$ & Local Standard of rest \\
HELIOCEN $\ldots \ldots$. & Relative to the Sun \\
BARYCENT $\ldots \ldots$ & Solar system barycenter \\
GEOCENTR $\ldots \ldots$ & Center of mass of the Earth \\
TOPOCENT $\ldots \ldots$ & Uncorrected \\
\hline \hline
\end{tabular}

\subsection{OI_TARGET (Revision 1) Specifications}

The specifications for the OI_TARGET table are given in Table 3.

\subsubsection{Target Position}

The RAEP0 and DECEP0 columns contain the right ascension and declination, respectively, of the phase center at the standard mean epoch, in degrees. RA_ERR and DEC_ERR contain the $1 \sigma$ uncertainties in these quantities. The phase center is assumed to be the pointing center. The EQUINOX field contains a (floating point) Julian year, giving both the epoch of the position (RAEP0 and DECEP0) and the equinox for the celestial coordinate system in which the position is expressed. The PMRA and PMDEC columns should contain the proper motions of the source in right ascension and declination, respectively, in degrees per Julian year. If the proper motion is unknown, then both fields should be set to zero. PMRA_ERR and PMDEC_ERR contain the $1 \sigma$ uncertainties in these quantities. If an apparent position at the time of an observation is required, it should be obtained by applying the appropriate transformations to the catalog position given by RAEP0 and DECEP0, making use of PMRA, PMDEC, and PARALLAX.

\subsubsection{Velocity Information}

The SYSVEL column gives the systemic radial velocity of the target (positive if receding). The VELTYP column contains a string that specifies the frame of reference for the systemic velocities. The string is one of the terms given in Table 4 . 
TABLE 5

OI_WAVELENGTH (REVISION 1)

\begin{tabular}{ccc}
\hline \hline Label & Data Type & Description \\
\hline \multicolumn{4}{c}{ Keyword } \\
\hline OI_REVN $\ldots \ldots .$. & I & Revision number of the table definition \\
INSNAME $\ldots \ldots .$. & A & Name of detector, for cross-referencing \\
\hline \multicolumn{2}{c}{ Column Headings (one row for each wavelength channel) } \\
\hline EFF_WAVE $\ldots . .$. & E (1) & Effective wavelength of channel $(\mathrm{m})$ \\
EFF_BAND $\ldots . .$. & E (1) & Effective bandpass of channel $(\mathrm{m})$ \\
\hline \hline
\end{tabular}

\subsection{OI_WAVELENGTH (Revision 1) Specifications}

The specifications for the OI_WAVELENGTH table are given in Table 5.

\subsubsection{Name of Detector}

Each OI_WAVELENGTH table in a file must have a unique value for INSNAME.

\subsubsection{Wavelengths}

Each OI_WAVELENGTH table describes the spectral response of detector(s) with a number of spectral channels. Each table gives the wavelengths for one or more of the data tables (OI_VIS, OI_VIS2, or OI_T3) and will often correspond to a single physical detector.

The EFF_WAVE column contains the best available estimate of the effective wavelength of each spectral channel, and the EFF_BAND column contains the best available estimate of the effective half-power bandwidth. These estimates should include the effect of the Earth's atmosphere, but not the spectrum of the target (the effect of the target spectrum should be taken into account as part of any model-fitting/mapping process; i.e., the target spectrum is part of the model).

\subsection{OI_VIS, OI_VIS2, and OI_T3 (Revision 1) Specifications}

The following comments apply to one or more of the OI_VIS, OI_VIS2, and OI_T3 tables (Tables 6, 7, and 8).

1. Cross-referencing.-Each data table must refer (via the INSNAME keyword) to a particular OI_WAVELENGTH table describing the wavelength channels for the measurements. Each data table may optionally refer, via the ARRNAME keyword, to an OI_ARRAY table.

2. Start date of observations.-This is a UTC date in the format YYYY-MM-DD (e.g., 1997-07-28).

3. Time of observation.-The value in the TIME column is the mean UTC time of the measurement in seconds since $0 \mathrm{hr}$ on DATE-OBS. Note that this may take negative values, or values $>86,400 \mathrm{~s}$, and hence the epoch of observation for the particular table is not restricted to DATE-OBS.

The value in the MJD column is the mean UTC time of the measurement, expressed as a modified Julian Date. It might be appropriate to use the MJD values instead of the TIME values when dealing with long time spans, but the standard makes no stipulation in this regard.

4. Integration time.-The exchange format will normally be used for interchange of time-averaged data. The "integration time" is therefore the length of time over which the data were averaged to yield the given data point.

5. Data arrays. - If the triple-product amplitudes are meaningless, as is the case for COAST data, NULL values for

TABLE 6

OI_VIS (REVISION 1)

\begin{tabular}{|c|c|c|}
\hline Label & Data Type & Description \\
\hline OI_REVN ......... & I & Revision number of the table definition \\
\hline DATE-OBS ....... & A & UTC start date of observations \\
\hline ARRNAME ........ & A & (Optional) Identifies corresponding OI_ARRAY \\
\hline INSNAME .... & A & Identifies corresponding OI_WAVELENGTH table \\
\hline \multicolumn{3}{|c|}{ Column Headings (one row for each measurement) } \\
\hline TARGET_ID ....... & I (1) & Target number as index into OI_TARGET table \\
\hline TIME ............. & $\mathrm{D}(1)$ & UTC time of observation (s) \\
\hline MJD .............. & $\mathrm{D}(1)$ & Modified Julian Date \\
\hline INT_TIME ......... & $\mathrm{D}(1)$ & Integration time (s) \\
\hline VISAMP $\ldots \ldots \ldots \ldots$ & D (NWAVE) & Visibility amplitude \\
\hline VISAMPERR & D (NWAVE) & Error in visibility amplitude \\
\hline VISPHI $\ldots \ldots \ldots$ & D (NWAVE) & Visibility phase in degrees \\
\hline VISPHIERR ... & D (NWAVE) & Error in visibility phase in degrees \\
\hline UCOORD ..... & D (1) & $u$ coordinate of the data $(\mathrm{m})$ \\
\hline VCOORD $\ldots \ldots \ldots$ & $\mathrm{D}(1)$ & $v$ coordinate of the data $(\mathrm{m})$ \\
\hline STA_INDEX ....... & I (2) & Station numbers contributing to the data \\
\hline FLAG $\ldots \ldots \ldots \ldots$ & L (NWAVE) & Flag \\
\hline
\end{tabular}


TABLE 7

OI_VIS2 (REVISION 1)

\begin{tabular}{|c|c|c|}
\hline Label & Data Type & Description \\
\hline \multicolumn{3}{|r|}{ Keywords } \\
\hline OI_REVN . & I & Revision number of the table definition \\
\hline DATE-OBS & A & UTC start date of observations \\
\hline ARRNAME $\ldots . . .$. & A & (Optional) Identifies corresponding OI_ARRAY \\
\hline INSNAME $\ldots \ldots .$. & A & Identifies corresponding OI_WAVELENGTH table \\
\hline \multicolumn{3}{|c|}{ Column Headings (one row for each measurement) } \\
\hline TARGET_ID ...... & I (1) & Target number as index into OI_TARGET table \\
\hline TIME ............. & $\mathrm{D}(1)$ & UTC time of observation (s) \\
\hline MJD $\ldots \ldots \ldots \ldots \ldots$ & $\mathrm{D}(1)$ & Modified Julian Date \\
\hline INT_TIME ........ & D (1) & Integration time (s) \\
\hline VIS2DATA $\ldots \ldots \ldots$ & D (NWAVE) & Squared visibility \\
\hline VIS2ERR .......... & D (NWAVE) & Error in squared visibility \\
\hline UCOORD ......... & $\mathrm{D}(1)$ & $u$ coordinate of the data $(\mathrm{m})$ \\
\hline VCOORD .......... & $\mathrm{D}(1)$ & $v$ coordinate of the data $(\mathrm{m})$ \\
\hline STA_INDEX ...... & I (2) & Station numbers contributing to the data \\
\hline FLAG $\ldots \ldots \ldots \ldots$ & L (NWAVE) & Flag \\
\hline
\end{tabular}

T3AMP may be used. The closure phases should still be treated as valid.

NWAVE is the number of distinct spectral channels recorded by the single (possibly "virtual") detector, as given by the NAXIS2 keyword of the relevant OI_WAVELENGTH table.

6. Complex visibility and visibility-squared uv coordinates.-UCOORD, VCOORD give the coordinates in meters of the point in the UV plane associated with the vector of visibilities. The data points may be averages over some region of the UV plane, but the current version of the standard says nothing about the averaging process. This may change in future versions of the standard.

7. Triple-product uv coordinates.-The U1COORD, V1COORD, U2COORD, and V2COORD columns contain the coordinates of the bispectrum point (see $\S 4$ for details). Note that U3COORD and V3COORD are implicit.

The corresponding data points may be averages in (bi-) spatial frequency space, but this version of the standard does not attempt to describe the averaging process.

8. Flag.- If a value in this vector is true, the corresponding datum should be ignored in all analyses.

TABLE 8

OI_T3 (REVISION 1)

\begin{tabular}{|c|c|c|}
\hline Label & Data Type & Description \\
\hline \multicolumn{3}{|r|}{ Keywords } \\
\hline OI_REVN .. & I & Revision number of the table definition \\
\hline DATE-OBS . & A & UTC start date of observations \\
\hline ARRNAME ...... & A & (Optional) Identifies corresponding OI_ARRAY \\
\hline INSNAME $\ldots \ldots .$. & A & Identifies corresponding OI_WAVELENGTH table \\
\hline \multicolumn{3}{|c|}{ Column Headings (one row for each measurement) } \\
\hline TARGET_ID ...... & I (1) & Target number as index into OI_TARGET table \\
\hline TIME $\ldots . . . \ldots \ldots .$. & $\mathrm{D}(1)$ & UTC time of observation (s) \\
\hline MJD $\ldots \ldots \ldots \ldots \ldots$ & $\mathrm{D}(1)$ & Modified Julian Date \\
\hline INT_TIME ....... & $\mathrm{D}(1)$ & Integration time $(\mathrm{s})$ \\
\hline T3АMP ....... & D (NWAVE) & Triple-product amplitude \\
\hline T3AMPERR & D (NWAVE) & Error in triple product amplitude \\
\hline Т3PHI ........ & D (NWAVE) & Triple-product phase in degrees \\
\hline T3PHIERR ....... & D (NWAVE) & Error in triple product phase in degrees \\
\hline U1COORD ........ & D (1) & $u$ coordinate of baseline $\mathrm{AB}$ of the triangle $(\mathrm{m})$ \\
\hline V1COORD ........ & $\mathrm{D}(1)$ & $v$ coordinate of baseline $\mathrm{AB}$ of the triangle $(\mathrm{m})$ \\
\hline U2COORD ........ & $\mathrm{D}(1)$ & $u$ coordinate of baseline $\mathrm{BC}$ of the triangle $(\mathrm{m})$ \\
\hline V2COORD ........ & $\mathrm{D}(1)$ & $v$ coordinate of baseline $\mathrm{BC}$ of the triangle $(\mathrm{m})$ \\
\hline STA_INDEX ...... & I (3) & Station numbers contributing to the data \\
\hline FLAG $\ldots \ldots \ldots \ldots$ & L (NWAVE) & Flag \\
\hline
\end{tabular}




\subsection{Optional Tables}

It may be useful to allow for some optional tables. For example, there might be one that contains instrument-specific information, such as the back-end configuration. Another optional table could contain information relevant to astrometry. The EXTNAMEs of additional tables should not begin with "OI_".

Development of the format was performed under the auspices of the IAU Working Group on Optical/IR Interferometry, and has the strong support of its members. We would like to thank Peter R. Lawson (Chair of the Working Group) for his encouragement and support.

The authors would like to thank David Buscher, Christian Hummel, and David Mozurkewich for providing material for the format specification and the OIFITS Web site. We would like to take this opportunity to thank all those in the community who have contributed to the definition of the format and its adoption by many interferometer projects.

\section{REFERENCES}

Cotton, W. D., et al. 1990, Going AIPS: A Programmer's Guide to the NRAO Astronomical Image Processing System (Charlottesville: NRAO), chap. 14.7, http://www.aoc.nrao.edu/aips/goaips.html

Flatters, C. 2000, The FITS Interferometry Data Interchange Format (AIPS Memo 102; Charlottesville: NRAO)

Hanisch, R. J., Farris, A., Greisen, E. W., Pence, W. D., Schlesinger, B. M., Teuben, P. J., Thompson, R. W., \& Warnock, A. 2001, A\&A, 376,359

Hummel, C. A., Pauls, T. A., \& Mozurkewich, D. 2002, Representation of Complex Visibilities (Cambridge: Univ. Cambridge), http://www. mrao.cam.ac.uk/\%7Ejsy1001/exchange/complex/complex.html
Lawson, P. R., et al. 2004, Proc. SPIE, 5491, 886

McAlister, H., \& Cornwell, T., ed. 2000, Report on the Workshop on Imaging with Ground-Based Optical Interferometers (Socorro: NSF), http://olbin.jpl.nasa.gov/papers/Report1.0.PDF

Monnier, J., et al. 2004, ApJ, 605, 436

Pauls, T. A., Young, J. S., Cotton, W. D., \& Monnier, J. D. 2004, Proc. SPIE, 5491, 1231

Thompson, A. R., Moran, J. M., \& Swenson, G. W., Jr. 1986, Interferometry and Aperture Synthesis in Radio Astronomy (New York: Wiley) 